\title{
Artificial Consciousness of a Hedonist and Its Control under Limits of Growth
}

\author{
V. Tsyganov \\ Department of Active Systems Theory, Institute of Control Sciences Russian Academy of Sciences, 65 Profsoyuznaya, \\ Moscow, 117997, Russia \\ *Corresponding Author: av188958@akado.ru
}

Copyright $(2014$ Horizon Research Publishing All rights reserved.

\begin{abstract}
Limits of global economic growth are negatively influencing on people level of life that leads to social instability. To consider this problem, psycho physiological model of artificial consciousness of a farseeing hedonist as a member of society is developed and investigated. It is shown that human nature of consumer produces constantly growing desire. After reaching the limits of growth, people from consumer society feel persistent negative emotions and dissatisfaction. It provides the problem of socio-economic stagnation. Its solutions can be increasing of demand (e.g., as a result of new Kondratieff technological cycle), or decreasing a consumption followed by its growth (e.g., global financial crisis or decay of state unions). Alternative humanistic solution is replacing the consumer values to the spiritual.
\end{abstract}

Keywords Artificial Consciousness, Intuition, Human, Psychology, Physiology, Hedonism, Emotion, Farseeing, Limits of Growth, Economic, Consumption, Liberalization, Stagnation, Control, Evolution, Spiritual

\section{Introduction}

The limits of global growth are due to limited natural resources and capacity of the biosphere [1]. The global financial crisis threatens economic stagnation and the crisis of the ideology of consumer society. Members of the post-industrial society of developed countries have traditionally focused on the growth of consumer. So they fear of falling living standards and socio-economic stagnation. This is indicated by the growing mass protests in Europe and USA. As pointed out by European political leaders (such as French Ex-President Nicolas Sarkozy), need to reform the socio-economic system. Such reform should strength people in terms of accelerating the dramatic changes. That reform fills their lives with new sense, historical optimism.

Activate the "human factor" enable advanced technologies of high tech evaluation and control of the human condition. On this basis, in recent years have been developed high human technologies - High Hume [2]. They should lie in background of new global technological structure, corresponding to the expected VI Kondratieff technological cycle. High Hume includes methods, approaches, and techniques to ensure coordination of private and public interests for development at rapid changes. Already, High Hume is implemented for improving the national security [3]. This paper focuses on the development of High Hume on the background of artificial consciousness to control evolution of a large scale socio-economic system under limits of global growth. The basis of such High Hume consists of comprehensive studies of human psychology and physiology.

\section{Artificial Consciousness of Farseeing Hedonist}

To investigate any socio-economic problem, we need to have model of consciousness and emotion of a member of society. It is widely used model of the rational "economic man", whose origins date back to Adam Smith and Karl Marx. Based on it, models of a farseeing element have traditionally been considered. This is model of homo sapiens, rationally using the available opportunities to achieve current and future goals. Since the beginning of this century this model was developed to take into account human psychology. In this model, called "Pupil", rationality and intuition is combined [2].

The theory states that the more adequate model to reality, the more effective control can be arranged. To construct a more adequate model of human behavior, the traditional model of farseeing element was subjected to further transformations including artificial consciousness and intuition. According to the doctrine of psychological hedonism, human actions are caused by the desire to obtain pleasure. If so, human is managed by desire. Then to provide a more adequate human model it is necessary to take into account its psychophysiology. The latest high technologies allow more accurately assess the condition of the person and, therefore, provide a basis for research and development of 
artificial consciousness and emotion models. Therefore, the next step is to construct a model of human behavior which combines rational thinking with feelings and emotionality.

Thus traditional model of farseeing element is complemented by two signal nervous systems. The first of these relates to the reaction of the autonomic nervous system (ANS), the second - to emotional reaction of the central nervous system (CNS). Supplementing their system of rational decision-making, which is related to artificial intelligence, find a hierarchical structure of the Farseeing Hedonist (briefly - FH), as shown in fig.1. In this structure, the low-level block - AFSS refers to ANS, ASSS - to CNS. The upper-level block AI corresponds to the artificial intelligence of FH. Both ASSS and AI are referred to Artificial Consciousness of FH. The output of AFSS is sensation $Y_{t}$. Also here $u_{t}$ - input, $N_{t}$ - primary physiological AFSS norm of sensation, $t$ - number of period, $t=0,1 \ldots$ The output of ASSS is emotion. There $y_{t}$ and $n_{t}$, are respectively state and norms of ASSS.

Suppose that in period $t$ on the input of AFSS acts stimulus:

$$
S_{t}=\left\{\begin{array}{l}
s>0, \text { if } t \leq \tau \leq t+\tau_{s} \\
0, \text { if } \tau<t \text { or } \tau>t+\tau_{s}
\end{array}\right.
$$

The solid line in fig.2a shows typical initial reaction $Y_{t}$ to stimulus $s_{t}$ in period $t=0$ in the form of positive sensation (briefly: +sensation) $Y_{t}$. It has a bell-shaped and characterized by the amplitude and duration of sensation. The sqlid line in fig. $2 \mathrm{~b}$ shows the secondary integral reaction $y_{t}=\sum Y_{\tau}$ to a stimulus $s_{t}$, in form of logistic curve. The dotteff line in fig. $2 \mathrm{~b}$ shows the secondary integral norms ( $n_{t}$ $\left.=\sum_{\tau=0} N_{\tau}\right)$. Initial FH reaction refers to the work of AFSS.

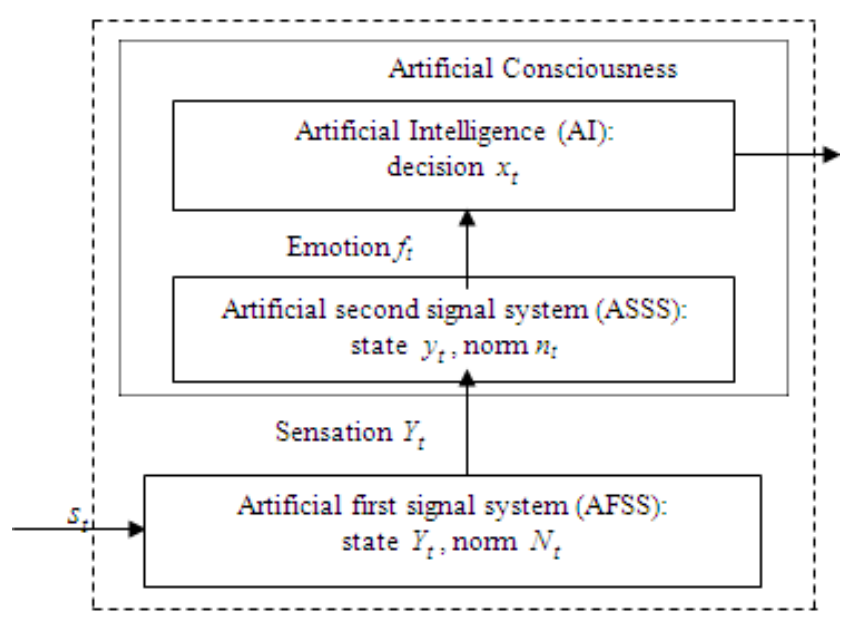

Figure 1. Artificial Consciousness of Farseeing Hedonist

The dependence of the primary $\mathrm{FH}$ reaction of the stimulus, as shown in fig.2a, is typical of the physiological reactions of ANS, such as galvanic skin response [2]. There $Y_{t}$ is skin-sympathetic evoked potential, measured in microvolts, $t$ - time in milliseconds. Therefore, the primary FH reaction can be considered analogous to the autonomic physiological reactions. Initially, the excitation increases rapidly, due to the functioning of the sympathetic nervous system. Then turn inhibitory (parasympathetic) nervous system. The end of the stimulus action leads to a damping of the primary reaction during the time $\tau_{k}$ (latency). With repeated stimuli (1), amplitude $Y_{t}$ and latency $\tau_{k}$ decrease, due to adaptation of ANS.

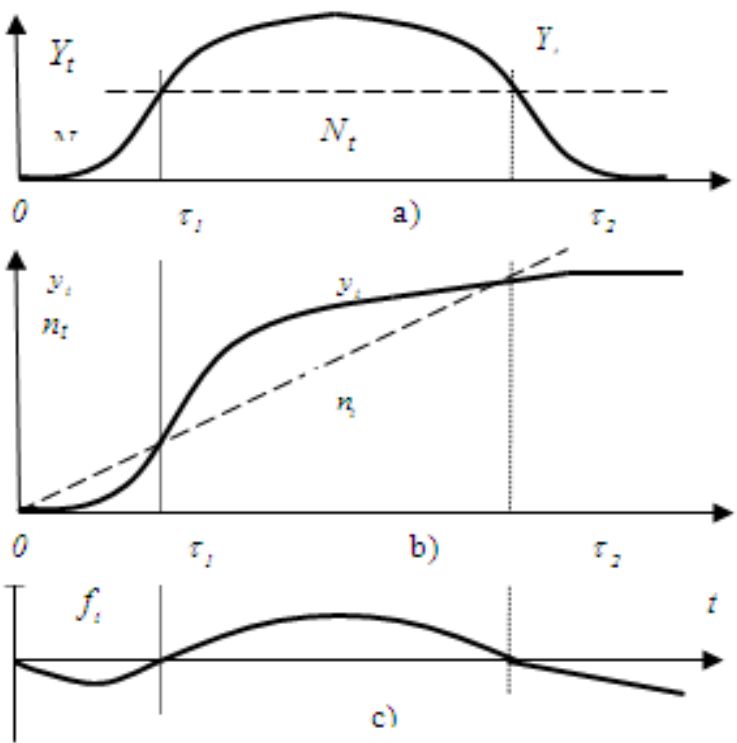

Figure 2. First (a) and integral (b) FH reaction at stimulus $s_{t}$; c) FH emotion

Integral response relates to the work of ASSS, bringing together information on past and current primary reactions. The dependence of the integral FH reaction to stimulus $s_{t}$ is shown in fig.2. It is typical emotional reactions of the CNS cognitive block responsible for programming, regulating and controlling the activities of the brain. Cognitive neurophysiologic apparatus uses a block prediction and evaluation of behavior, and correlates them with the standards stored in associative memory [2]. The reaction of the cognitive unit, combining (integrating) ANS reaction, and compare them with previous experience, relate to the integrated FH reaction (fig.2b). Therefore, the integrated response of the FH (and its parameters) is called cognitive. Thus, the primary FH reaction is related to physiological reaction of the ANS, and cognitive reaction - to emotional reaction to the CNS. In practice, physiological and emotional reactions of the individual measured and modeled with the help of modern psycho physiological technologies, such as devices operating on the principle of polygraph - "lie detector" [2].

The activity is expressed in the individual psyche needs a certain level (norm $N_{t}$ ) of the sensation $Y_{t}$ in each time period. The dotted lines in fig.2 show the primary physiological $\left(N_{t}\right)$ and cognitive psychological $\left(n_{t}\right)$ norms. Stimulus (1) cause the +sensation and form a favorable stationary situation (briefly: f-situation) for FH. Medium, bringing together all sorts of +situations, is called favorable medium (briefly: f-medium).

Human response to external stimuli is expressed in 
emotions. In line with modern concepts of psychophysiology, emotions play a role of a criterion of utility, the "universal currency", which determines the value of the stimulus [2]. Positive emotions (briefly, +emotion) reflect the beneficial effects, negative emotions (-emotion) - harmful. It is assumed that the strength and sign of emotion $f_{t}$ in period $t$ depends on the cognitive states $y_{t}$ and norms $n_{t}$ :

$$
f_{t}=F\left(n_{t}, y_{t}\right)=\left\{\begin{array}{l}
F\left(n_{t}, y_{t}\right)>0, \text { if } y_{t}>n_{t}, \\
F\left(n_{t}, y_{t}\right)<0, \text { if } y_{t}<n_{t}
\end{array}\right.
$$

Here $F\left(n_{t}, y_{t}\right)$ is emotional function. If the state exceeds the norm $\left(y_{t}>n_{t}\right)$ then emotion is positive $\left(F\left(n_{t}, y_{t}\right)>0\right)$, otherwise (if $\left.y_{t}<n_{t}\right)$-negative $\left(F\left(n_{t}, y_{t}\right)<0\right)$. If the status is normal $\left(y_{t}=n_{t}\right)$, there is no emotion ("zero emotion": $F\left(n_{t}, n_{t}\right)=0$ ). Emotional function (2) can be determined by experimental psycho physiological methods [2].

Z.Freud assigned an important role in psychoanalysis for a life instinct. It is associated with the hope of getting +emotion into the future. On the basis of this instinct, it is possible to form an understanding of the sense of FH life associated with such hope. Formally, it represents next

Definition 1. We shall say that Farseeing Hedonist life has a sense if:

$$
\forall t \exists \tau \text { such that } \tau \geq t \text {, and } F\left(n_{\tau}, y_{\tau}\right)>0
$$

If (3) is not satisfied, we shall say that his life has no sense.

\section{Hedonism and Stagnation}

Post-industrial society should create a favorable inpatient environment for its members. It seems that FH life in such an environment is associated with +emotions and has a sense. Paradoxically, however, but fair

Theorem 1. Farseeing Hedonist life has no sense in the favorable stationary situation.

PROOF. Primary and integrated response to a stimulus $s_{t}$, as well as FH emotions $f_{t}$, shown in fig.2. As seen in fig.2b,c, for sufficiently large $t, y_{t}<n_{t}$ and $F\left(n_{t}, y_{t}\right)<0$. Thus, there is no $\tau$ such that $\tau \geq t$, and $F\left(n_{\tau}, y_{\tau}\right)>0$ takes place. Consequently, condition (3) does not hold. So accordingly to definition 1, FH life has no sense, QED.

Substantially, the senselessness of Farseeing Hedonist life is due to lack changes leading to new pleasures in stationary supportive environment. The paradox of Theorem 1 is formally reflects the problem of social stagnation - a contradiction between the pursuit of hedonistic pleasure in a hospital environment and the need of change to prevent the loss of life sense. In fact, the problem of social stagnation is related to satiety hedonist serene life. Subjectively, the lack of life sense in inpatient environment can be considered as regress, and motivate people to action. Their frustration and aggression may apply to other people, society, government, existing order.

Fig.2c shows that the +emotions related to the satisfied FH desire, eventually pass. "Everything pass", - said King
Solomon. Therefore, the changes are needed for FH, followed by its new +sensations and +emotions.

Definition 2. Let's talk about the regular change of the situation if, for any period $t$, there exists future finite period $v$ (i.e. $v \geq t$ ), in which stimulus $s_{v}$ defined by (1) occurs at the entrance of $\mathrm{FH}$.

Theorem 2. To have sense of Farseeing Hedonist life in favorable medium it is necessary and sufficient to change the situation regularly.

PROOF. Necessity is proved by contradiction. There are only favorable stationary situations in a favorable medium. Let us assume that $\mathrm{FH}$ life has a sense when situation remains unchanged since period $t_{0}, t_{0}=0,1, \ldots$ Then the primary and integrated response to a stimulus $s_{t}$, as well as emotions $f_{t}$ are shown in fig.2. After a period of $t_{0}+\tau_{2}$, there is $n_{t}>y_{t}$, and $\mathrm{FH}$ feels -emotions. However, the condition of Theorem 2 said about presence sense of FH life. According to (3), it means that exists $\tau$ such that $\tau \geq t$, and $F\left(n_{\tau}, y_{\tau}\right)>0$. Consequently, the assumption of the immutability of the situation contradicts the condition of Theorem 2. This contradiction proves the necessity.

Now let us prove sufficiency. If situation is regularly changed, then by definition 2, for any period $t$ exists $v$ such that $v \geq t$, and in the period $v \mathrm{FH}$ occurs the new input stimulus. Then $y_{t}>n_{t}$ during the time interval $\left(v+\tau_{1}, v+\right.$ $\left.\tau_{2}\right)$. So for any $t$ such $v$ exists that $v \geq t$, and $F\left(n_{v}, y_{v}\right)>0$. Therefore, according to definition 1, FH life has a sense, which proves sufficiency, QED.

Meaningful, regular change of scenery will initiate new stimuli, leading to obtain new +emotions and giving sense of FH life. Thus Theorem 2 is theoretically justifies the traditional recipe for depression - a change of scenery. In large scale socio-economic systems, it proves the old as the hills, the motto of politicians: "Bread and circuses!"

\section{Stairs Desires}

By Theorem 2, regular changes of scenery give sense of FH life in a supportive environment. They leads to an emotional series - the temporal sequence of positive and negative emotions ("white" and "black" strips a person's life). Such unlimited sequence formed by a chain of stimuli (1) is called Stairs Desires (SD). Obviously, to realize SD in favorable media it is necessary and sufficient to change situation regularly. Fig. 3 shows the primary (a) and integral (b) FH reaction on such stimuli sequence, as well as appropriate $\mathrm{FH}$ emotions (c).

Theorem 3. To give Farseeing Hedonist life sense in a favorable stationary media, it is necessary and sufficient to realize the stairs of his desires.

PROOF. By Theorem 2, to give sense of FH life in f-media, it is necessary and sufficient to change situation regularly. But that means realization of SD by definition, QED.

In other words, realizing the SD, FH can always rely on the +emotion into the future, and his life becomes meaningful. 


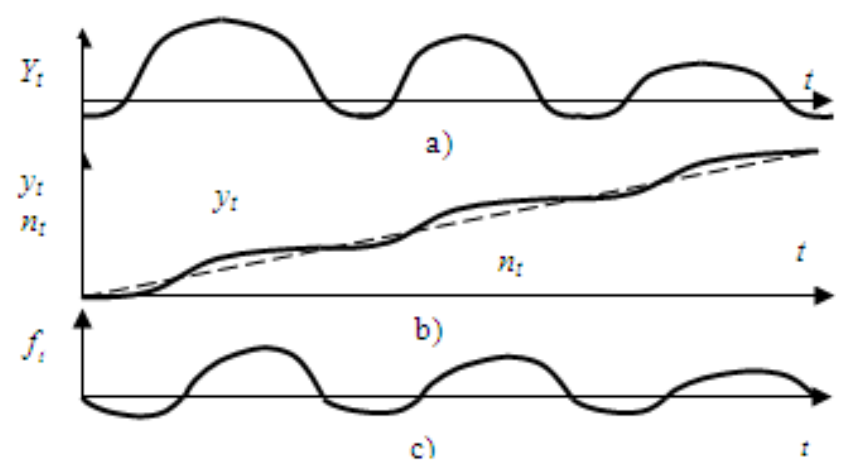

Figure 3. Stairs Desires of Farseeing Hedonist

Definition 3. Farseeing Hedonist, self-forming Stairs Desires by changing the situation, called the Creative FH (briefly - CFH).

Corollary 1. Life of a Creative Farseeing Hedonist has a sense in the favorable medium.

PROOF. According to Theorem 3, to give FH life sense it is necessary and sufficient to realize SD. By definition 3, CFH realizes SD. So, life CFH has a sense, QED.

Note that by Theorem 1, life sense of passively Farseeing Hedonist in favorable environment is absent, and it provides depression. In accordance with the Pareto principle, society is composed of $80 \%$ of passive personality, and by $20 \%$ - of the creative individuals (creators). Then, by Corollary 1, to avoid a depression in the community, located in f-medium, it is enough to turn passive personalities in creators. This formed the basis of progressivism - type of hedonism, the ideology for development to meet the ever growing desire. For example, the desire to increase capital leads to unlimited accumulation. Progressivism activates the creative individual in the socio-economic system contributing to the development of passive personality environment. Conversely, the creator transformation into passive personality leads to depression according to Theorem 1 . Changing the environment can be achieved as a result of purposeful activity of the individual himself, and then we talk about the active changes. If changes of scenery are determined by external factors, we speak about passive changes.

\section{Disintegration Problem}

A world ruled by desire generates an ideology of consumption. $\mathrm{CFH}$, whose values are associated with the consumption (briefly - the consumer-CFH) or accumulation (short - drive-CFH) can itself change the situation for the construction of his own SD. Formally, the state of consumer-CFH defined by the volume of consumption, the state of drive-CFH - by its capital. These quantitative measures of success are used in the consumer society and business. For example, state of "economic man" can be expressed in money. Assume that CFH consumption in period $t$ characterizes the value of $y_{t}$. Due to global growth limits, there is an objective restriction of $L$ (fig.4b).
The nature of CFH is that the consumer's needs (ambitions) $n_{t}$ are constantly growing (dashed line in fig.4b). Limit of growth of $L$ is reached in period $\tau_{L}$. Before this period, $\mathrm{CFH}$ desire of + emotions is satisfied from time to time, due to new consumption and relevant stimuli (fig.4a). So $y_{t}$ (solid wavy line in fig.4b) periodically over the growing ambitions of $n_{t}$, and then CFH is experiencing +emotions (fig.4c). The situation changed after reaching growth limit $L$. Beginning from time $\tau_{L}$, CFH experiences persistent -emotions (fig.4c). Thus, the objective stagnation of consumption contradicts to the growing of CFH needs $\left(n_{t}\right)$. In fact, the problem of stagnation (section 2) occurs in the sphere of consumption and capital accumulation. For brevity, we refer to it as a problem of economic stagnation. Such stagnation leads to depression both consumers-CFH and drives-CFH. Their anger and aggression may be paid to the social environment.

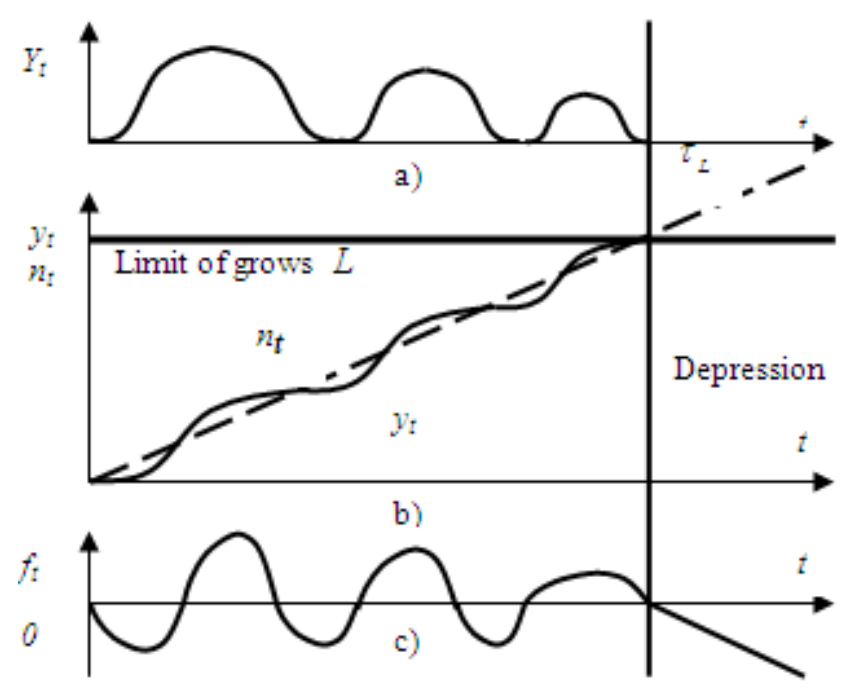

Figure 4. Feelings and emotions of the Creative Farseeing Hedonist under limits of growth

As shown in section 4, the problem of economic stagnation is due to the restrictions on consumption and capital (short - economic constraints) for the active, creative people, who themselves can change the socio-economic situation and realize their own SD (Corollary 1). But the economic constraints cause the limits of consumption. Nevertheless, desire of active, creative people is constantly growing. Periodically, such people are met by the new stimulus, and then they are experiencing +emotions. However, after reaching the limit of growth, they are replaced by -emotions that provides depression (fig.4).

Note that active people have the potential to change the economic environment for passive people. But due to economic constraints, active people have no possibility to improve economic conditions to passive people. The latter themselves can not change the situation. Therefore, due to limits of growth, the economic environment is stationary for a passive citizen. But then, by Theorem 1, the life of passive farseeing hedonist has no sense. Remember that according to the Pareto principle, in a society up to $80 \%$ of passive people. 
So loss of sense of their life leads to social stagnation. Thus, the problem of economic stagnation faced by an active minority, raises the problem of social stagnation related to the rest of society. Then it can talk about the problem of socio-economic stagnation. Above arguments lead to the conclusion that, because of its importance, can be formulated as

Proposition 1. Economic constraints provide the problem of socio-economic stagnation.

In fact, such stagnation is the result of steady-state socio-economic environment in which $\mathrm{FH}$ life has no sense (Theorem 1). To solve the problem of socio-economic stagnation, it is necessary to eliminate its causes. Given Proposition 1, this solution is associated with the removal of economic restrictions $(L)$. Traditional solution lies in expanding limits of growth (e.g., increasing demand which may be result of new Kondratieff technological cycle). Alternative solution is liberalization. Then the creative drive-CFH can itself change the situation, getting the +emotions and realizing SD. Furthermore, he obtains capital and can change the socio-economic environment. That provides the possibility for passive people to realize their SD and finally to avoid socio-economic stagnation.

In a high centralized (e.g., planning) system, economic restrictions $L$ are established by government. If these restrictions are acting for a long time, there will be socio-economic stagnation (fig.4). Then some people can blame the government ignoring objective reasons for restrictions (such as global limits to growth).This dissatisfaction can take advantage of the opponents of the government for its overthrow. In large scale socio-economic system, which consist of many relatively independent subsystems (such as the Soviet Union or European Union, as an alliance of partially independent states), this issue of stagnation raises the disintegration problem.

For example, socio-economic stagnation is considered the major cause of the disintegration of the Soviet Union. There was officially adopted communist ideology of unlimited consumption: "Taking from each man according to his ability, giving to each man according to his needs". USSR economy was formed around Russia, and many other peripheral economies (such as Georgia) have been weak and subsidized. In the middle of 1980 s, limits of growth and consumption felled with oil prices. Government has established direct economic constraints $L$ to Soviet Union republics consumption. Acting for a long time, they contributed to the socio-economic stagnation in the national republics and increased dissatisfaction with their elites.

Economic ambitions of people from these national republics were growing up, not corresponding to the whole USSR constraints. The most active representatives of local elites were accused central USSR government of the infringement of interests of small nation. To save USSR in these circumstances, it was necessary to weaken the mentioned economic constraints, i.e. to liberalize economies. In this case the formal constraints of consumption $L$ from the USSR government would be absent. Therefore, national elites of USSR republics were inclined to look for objective reasons of insufficient consumption and to eliminate them without blaming the central government. Such liberalization could support both the human desire for self-organization and stability of the government and USSR as a whole. But the weakening of economic restrictions should be done consistently and carefully. As in adaptive control it should be critically assess the impact of each step of liberalization, gradually adjusting the economies like in China. But he Soviet leadership did not have appropriate knowledge and experience and sharply refused economic constraints. Uncontrolled liberalization has led to chaos and disintegration of the USSR.

\section{Flexible Liberalization}

The basic contradiction of the socio-economic system is between the private and public interests. In our model it is transformed into a contradiction between economic constraints and the desire of +emotion for an active person (and, therefore, for a permanent changes). This contradiction may deprive sense of FH life and lead active person to depression, frustration, aggression, etc. In consequence can be excitement, disintegration, etc.

Consider solution of stagnation problem in stochastic growth limits $L_{t}$ (fig.5b). As before, it is supposed that the CFH consumption in period $t$ is characterized by $y_{t}$. According to Proposition 1, the economic constraint $C_{l}$ posed the stagnation problem before period $\tau_{L 1}$ (fig.5b). Liberalization in the period $\tau_{L I}$ removes limitation $C_{l}$. CFH desire are characterized by norm $n_{t}$ (dashed line in fig. $5 \mathrm{~b}$ ) periodically met by stimuli (fig.5a) associated with the growth of consumption or capital. So $y_{t}$ (wavy line on the fig.5b) periodically exceeds $n_{t}$, and CFH is experiencing + emotion (fig.5c). The situation changed in the period $\tau_{L 2}$, after $y_{t}$ reaching $L_{t}$. Then $\mathrm{CFH}$ is experiencing persistent -emotions, and problem of stagnation takes place. It can be solved centrally or spontaneously.

First of all, government may introduce in period $\tau_{L 2}$ new economic constraints $C_{2}$ (fig.5b). For example, devaluation or default reduces consumption and capital, after which government should stabilize the situation. Second, the collapse of consumption and accumulation can occur spontaneously, due to decline in demand or the global financial crisis (e.g. in 2008). In both these cases, $y_{t}$ is reduced to the level of $C_{2}$. After that, the government takes measures to stabilize the situation (for example, supports the economy after 2008). Strengthening the role of the government (centralization) stabilized the situation. The end stage of stabilization and central support marks the end of the cycle of evolution of the economic system, which includes periods of liberalization and centralization. After stabilization, since the period $\tau_{L 2}+1$, the government holds the newly liberalization, removing restrictions $C_{2}$ (fig. $5 \mathrm{~b}$ ). This causes a new +emotion of CFH (fig. 5 c) by new stimuli (fig.5a). The situation changed in the period $\tau_{L 3}$, after 
reaching the constraints for consumption due to new global limits (e.g., increased consumption in developing countries, such as BRICS). Again, there problem of stagnation can arise. It can be solved by a rapid decrease in consumption and capital $y_{t}$ to the level of $C_{3}$ and so on.

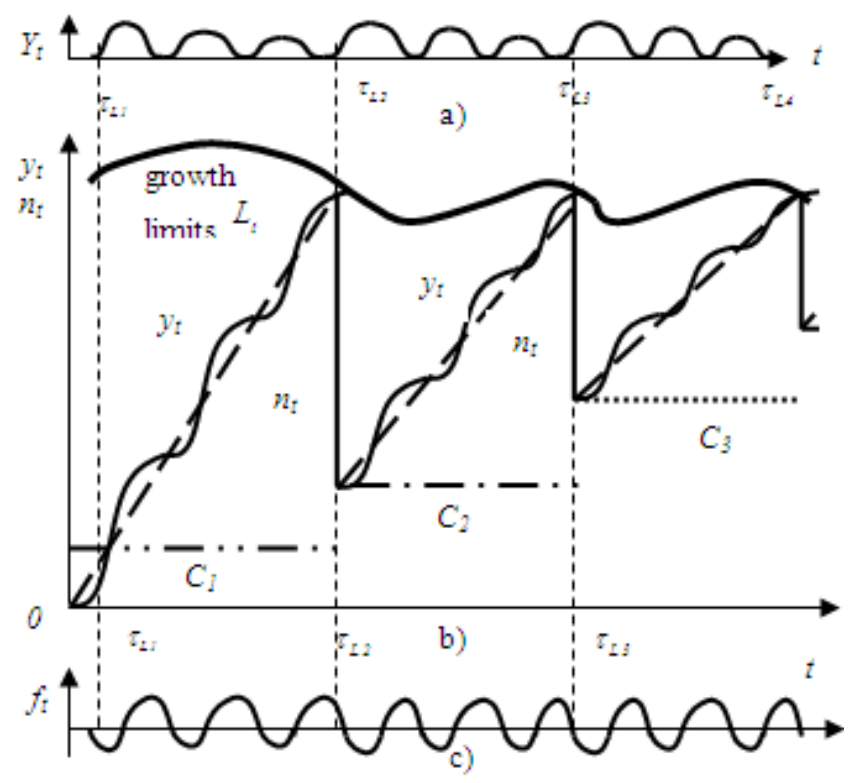

Figure 5. Flexible liberalization of Creative Farseeing Hedonist

The concept of flexible (adaptive) liberalization involves the removal of restrictions in a period of declining of economic activity. Then cyclical evolution of the economic system includes periods of growth and adjustment of consumption and accumulation (fig.5). Each cycle of liberalization and centralization provides cycle of capital savings and capital losses. From the other point of view, the cycle of fall and growth of consumption are realized. Repetition of these cycles helps to optimize the socio-economic system (in particular, when limit consumption $L_{t}$ is approaching), resolving the basic contradiction of consumer society - between intake desire and limits of growth.

\section{Adaptive Control of Evolution}

Liberalization solves the problem of socio-economic stagnation, supporting entrepreneurial initiative and self-organization of society. However, excessive desire growth of consumption and accumulation leads to problem of stagnation. Adaptive socio-economic governance is associated with periodic solving this problem.

The problems of socio-economic stagnation can be resolved, first, the onset of VI Kondratieff technological cycle in which the growth of consumption and capital takes place limiting or even reducing needed resources and pollutions (in this case limit $L_{t}$ increase). Second, it can be solved by decreasing of consumption, followed by its growth (e.g., global financial crisis or devaluation). Then the cycles of liberalization and centralization takes place (section 6).
Alternative humanistic solution is replacing the consumer values by the spiritual values. According to Corollary 1 , in order to avoid depression in the hospital environment it is enough to turn passive hedonist in a creator. He gets +emotions, fulfilling own spiritual desires, and implements his own SD. For example, in USSR posed the problem of mass education of talented people into spiritual creators. In practice, such replacement needs, first of all, a change in the consciousness of active people from a middle class. Because of their daily needs are mainly satisfied, replacement the consumer values by the spiritual ones is natural. Creative people, focused on spiritual values, do not need unlimited growing consumption and capital. In such a way, favorable medium provides middle class and new spiritual creators. For example, the Soviet leaders believed they could solve the problems of stagnation and disintegration due to unlimited spiritual growth. Unfortunately, most of USSR population do not belongs to middle class. So the sharp oil prices decline and falling standards of living have led to fiasco of this humanistic concept. Socio-economic stagnation led to the disintegration of the USSR (section 5). Note that $M$. Thatcher in UK and R. Reagan in USA solved a similar issue through liberal reforms, which provided the advantage of the West.

In modern times, European Union is facing with similar problems of stagnation and disintegration. This contributes to the implementing ideology of unlimited consumption in the EU, which includes some weak periphery national economies (like Greece). Therefore, it can not be excluded the possibility of decay of European Union, like in Soviet Union. It should decrease consumption in EU, followed by its growth (as it was in Russia after USSR decay). Spontaneous solutions maintaining the ideology of consumption are regular global financial crises (like in 2008). In this connection, it can be talk about the cycles of crisis. Global consumption reduction in 2008 had been achieved spontaneously due to global financial crisis $\left(\tau_{L 2}=2008\right.$, fig. 5 b). Consumption is expected to decline in $2014\left(\tau_{L 3}\right.$ $=2014$ on fig. 5 b) may be intentional - through the conscious restriction of consumption in some of 28 countries of the EU. Of course, it can not be excluded the natural decrease in consumption due to the new global crisis. The devaluation of Euro may also reduce consumption in the EU. However, if there will be continued domination the ideology of unlimited consumption, the governments will seek to increase consumption all costs. This will require resources from other countries, and give rise to aggressive ambitions, huge military budgets and wars for natural resources and markets.

\section{Conclusions}

Limits of global economic growth constraints people consumption. Investigation of the psycho physiological model of artificial consciousness of a farseeing hedonist is based on human nature produces constantly growing desire. After reaching the limits of growth, people from consumer 
society feel persistent negative emotions and dissatisfaction. It provides the problems of socio-economic stagnation and disintegration. Their main solutions are: expansion (e.g., increasing of demand as a result of new VI Kondratieff technological cycle), or decreasing consumption, followed by its growth (e.g., global financial crisis or devaluation). Alternative humanistic solution is replacing the consumer values the spiritual.

\section{REFERENCES}

[1] Kile F., and G. Dimirovski. Choices for Global Social Stability. In: Reports of the $17^{\text {th }}$ IFAC World Congress, 2008, pp. 6681-6685. Seoul.

[2] Tsyganov V. Adaptive Mechanisms \& High Hume: Theory of Human Systems. - Moscow, Academy Project Publishing, 2012 (in Russian).

[3] Schultz V., and V. Tsyganov. Modernization of National Security. - Moscow, Nauka Publishing, 2010 (in Russian). 